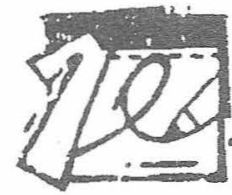

Educación Primaria

\title{
ECOMUSEO: UNA PROPUESTA METODOLÓGICA
}

\author{
Alfredo Miranda Calderón* \\ Elizabeth Alpizar Barrantes**
}

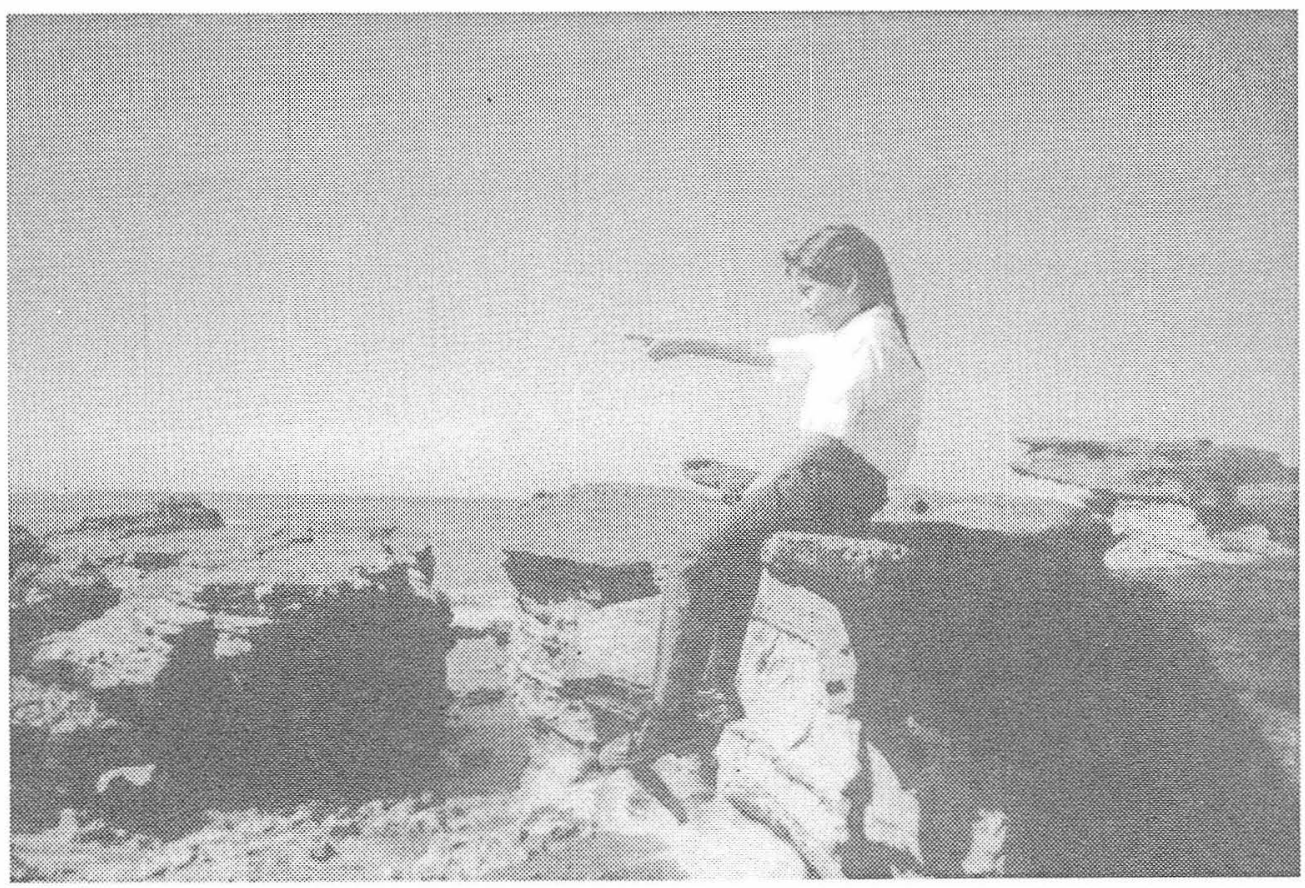

* Egresado de la Escuela de Formación Docente de la Facultad de Educación - UCR y del Programa de Licenciatura en I y II ciclo de la División de Educación Rural del CIDE- UNA.

Desde 1990 se ha desempeñado como maestro unidocente en el Centro Educativo Mal País. Dicha institución se localiza en el extremo sureste de la Península de Nicoya cerca de la Reserva Natural Absoluta Cabo Blanco.

** Licenciada en I y II Ciclos, graduada en la División de Educación Rural del CIDE-UNA. Actualmente se desempeña como maestra en la escuela Eulogio Salazar Lara en Sarchí. 
Este documento forma parte de un proyecto que pretende facilitar la meditación pedagógica de quienes enseñamos y aprendemos en escuelas unidocentes. Está dirigido a los maestros y maestras que por la localización y características de sus escuelas, deben compartir espacios que integran diversas edades, intereses y niveles académicos.

Para su elaboración se han tomado en cuenta las necesidades reales de los docentes así como la opinión de estudiantes de escuelas unidocentes.

El diseño de cada una de las actividades que comprende esta propuesta propicia el aprendizaje pertinente en un contexto natural, ya que toma en cuenta todos los elementos con que disponen los centros educativos de estas zonas. Asímismo las experiencias que se desarrollen incluyen la observación, la investigación y desarrollo de habilidades y destrezas.

Todas las actividades que a continuación sugerimos constituyen un medio para que los docentes que imparten varios grados simultáneamente introduzcan gradual e integralmente a sus estudiantes al conocimiento, comprensión y valoración de su contexto natural.
This article is part of a project intended to facilitate pedagogical meditation among all of us who teach and learn in one rooms one teacher schools. It is addressed to all teachers who must share classrooms comprised by students of different ages. interests and levels of competence. a situation that is brought about by the location an characteristics of their schools. Also, the real needs these teachers have, as well as the opinions of students attending one-teacher schools are also taken into account.

Each one of the activities conforming this proposal is designed to promote learning in a natural context, for they involve all the elements available at these schools. The experiences developed include observation. research and skill development.

All the activities suggested here serve as a meansfor teachers who teach different grades simultaneously so they can transmit to their students knowledge about. understanding and valuation of their natural surroundings gradually and in an holistic way: 


\section{¿CÓMO USAR ESTA GUÍA?}

Para facilitar la aplicación de las actividades se han establecido ciertos parámetros que describen el espacio donde se podrían desarrollar estas estrategias así comolas áreas del currículo que se integran. Dicha información se describe a continuación y aparece sugerida en las guías a través de íconos o símbolos informativos.

\section{ESPACIO DEL ECOMUSEO}

\begin{tabular}{|c|c|c|}
\hline Reominuseo & $\begin{array}{l}\text { Nombre del } \\
\text { mestordo }\end{array}$ & 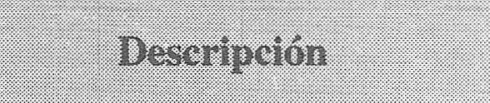 \\
\hline & $\begin{array}{c}\text { Ecomuseo } \\
\text { escolar }\end{array}$ & $\begin{array}{l}\text { Espacio escolar dedicado a la } \\
\text { experimentación, manipulación y } \\
\text { exhibición de proyectos individuales } \\
\text { o grupales. Su función es recopilar } \\
\text { y concretizar el conocimiento } \\
\text { adquirido por los estudiantes. }\end{array}$ \\
\hline & $\begin{array}{l}\text { Reserva } \\
\text { Natural }\end{array}$ & $\begin{array}{l}\text { Área o extensión destinada a la } \\
\text { conservación y protección de los } \\
\text { recursos naturales de la región. } \\
\text { Dichas instituciones constituyen un } \\
\text { aliado en cuanto a la consecución } \\
\text { de los fines del ecomuseo, ya que } \\
\text { se proyectan socialmente a las } \\
\text { comunidades a través de programas } \\
\text { ambientales. }\end{array}$ \\
\hline & Mini Reserva & $\begin{array}{l}\text { Extensiones de terreno propiedad de } \\
\text { la escuela o de algún vecino que son } \\
\text { utilizadas por los estudiantes para } \\
\text { poneren práctica actividades propias } \\
\text { del ecomuseo y el desarrollo de } \\
\text { prácticas ambientalistas. }\end{array}$ \\
\hline
\end{tabular}




\section{ÁREAS DEL CURRÍCULO}

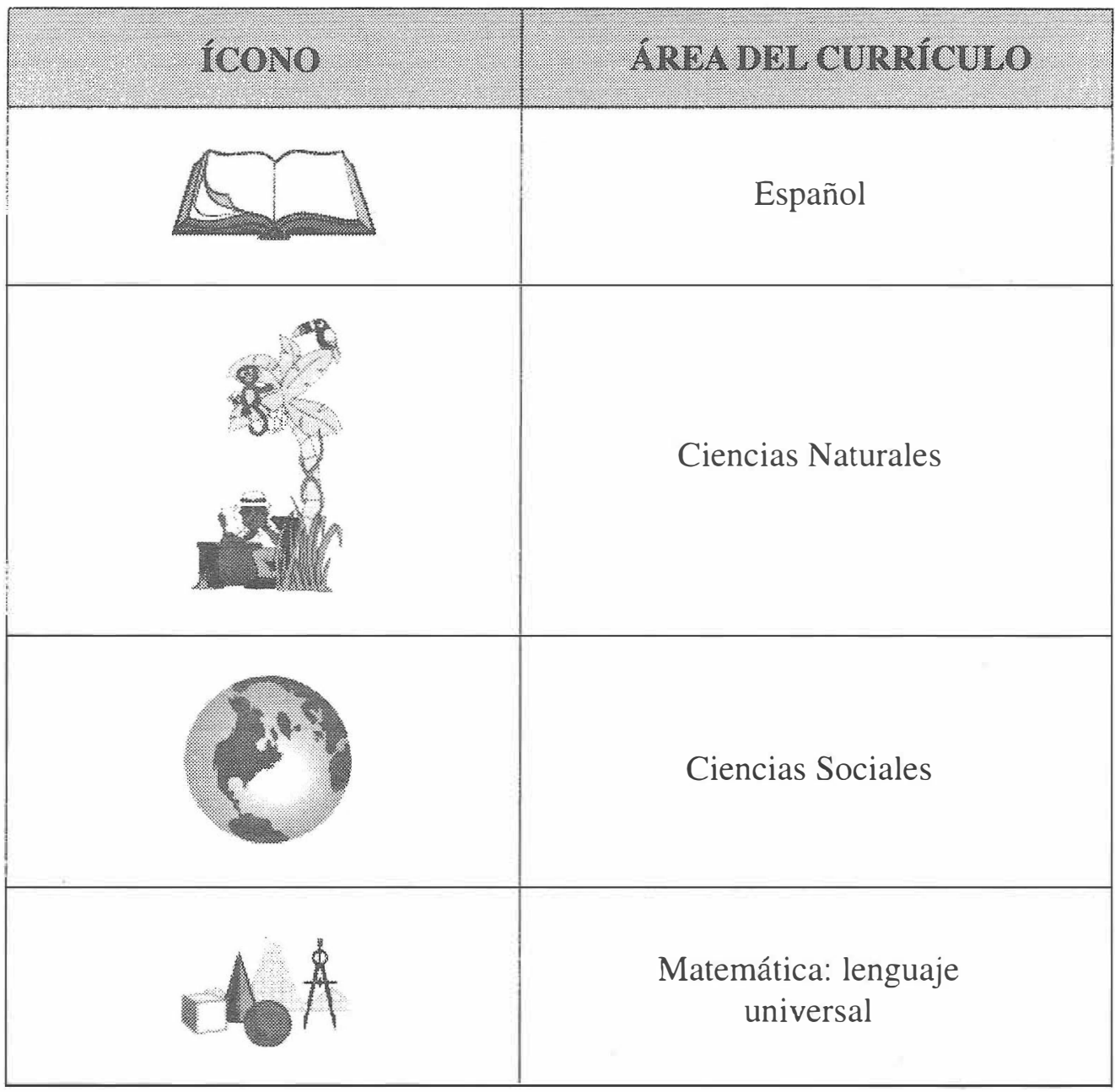

A continuación se presenta un ejemplo de la integración llevada a cabo entre las cuatro materias mencionadas. 


\section{REFERENCIA INTEGRADORA}

\begin{tabular}{|c|c|c|c|}
\hline $\begin{array}{l}\text { Matemática como } \\
\text { lenguaje universal }\end{array}$ & $\begin{array}{c}\text { Ciencias } \\
\text { Naturales }\end{array}$ & Comunicación & $\begin{array}{l}\text { Ciencias } \\
\text { Sociales }\end{array}$ \\
\hline CONTENIDOS & CONTENIDOS & CONTENIDOS & CONTENIDOS \\
\hline $\begin{array}{l}\text { - Estimación de cantidades } \\
\text { - Operaciones fundamentales } \\
\text { - Relaciones de orden } \\
\text { - Número ordinales y romanos } \\
\text { - Comparación de cantidades } \\
\text { - Medidas: longitud, tiempo, } \\
\text { masa y superficie } \\
\text { - Geometría: polígonos, } \\
\text { cuadriláteros, el triángulo, } \\
\text { circunferencia y círculo } \\
\text { - Cálculo de perímetro y áreas } \\
\text { - Estadística }\end{array}$ & $\begin{array}{l}\text { - Materia: tipos y clasificación } \\
\text { - energía: tipos, manifestaciones } \\
\text { y fuentes } \\
\text { - Ecología: niveles tróficos } \\
\text { - Relaciones simbióticas y } \\
\text { antagónicas } \\
\text { - Reinos biológicos } \\
\text { - Fotosíntesis } \\
\text { - Taxonomía } \\
\text { - Ecosistemas: componentes } \\
\text { bióticos y abióticos } \\
\text { - Desarrollo sostenible } \\
\text { - Manejo de desechos } \\
\text { - Procesos naturales }\end{array}$ & $\begin{array}{l}\text { - Elementos de la comunicación } \\
\text { - Morfología: sustantivos, } \\
\text { adjetivos, verbos } \\
\text { - Descripciones } \\
\text { - Figuras literarias } \\
\text { - Ideas principales y } \\
\text { complementarias } \\
\text { - Tipos de texto } \\
\text { - Antónimos y sinónimos } \\
\text { - La carta } \\
\text { - Ortografía: reglas ortográficas } \\
\text { y leyes del acento }\end{array}$ & $\begin{array}{l}\text { - Cartografía } \\
\text { - Coordenadas geográficas } \\
\text { - Localización geográfica } \\
\text { - Clima } \\
\text { - Estado del tiempo } \\
\text { - Zonas climáticas } \\
\text { - Costas: utilidad } \\
\text { - Aprovechamiento de recursos } \\
\text { naturales } \\
\text { - Relaciones causa-efecto } \\
\text { - Límites-ciases } \\
\text { - Mar territorial y mar } \\
\text { patrimonial }\end{array}$ \\
\hline
\end{tabular}



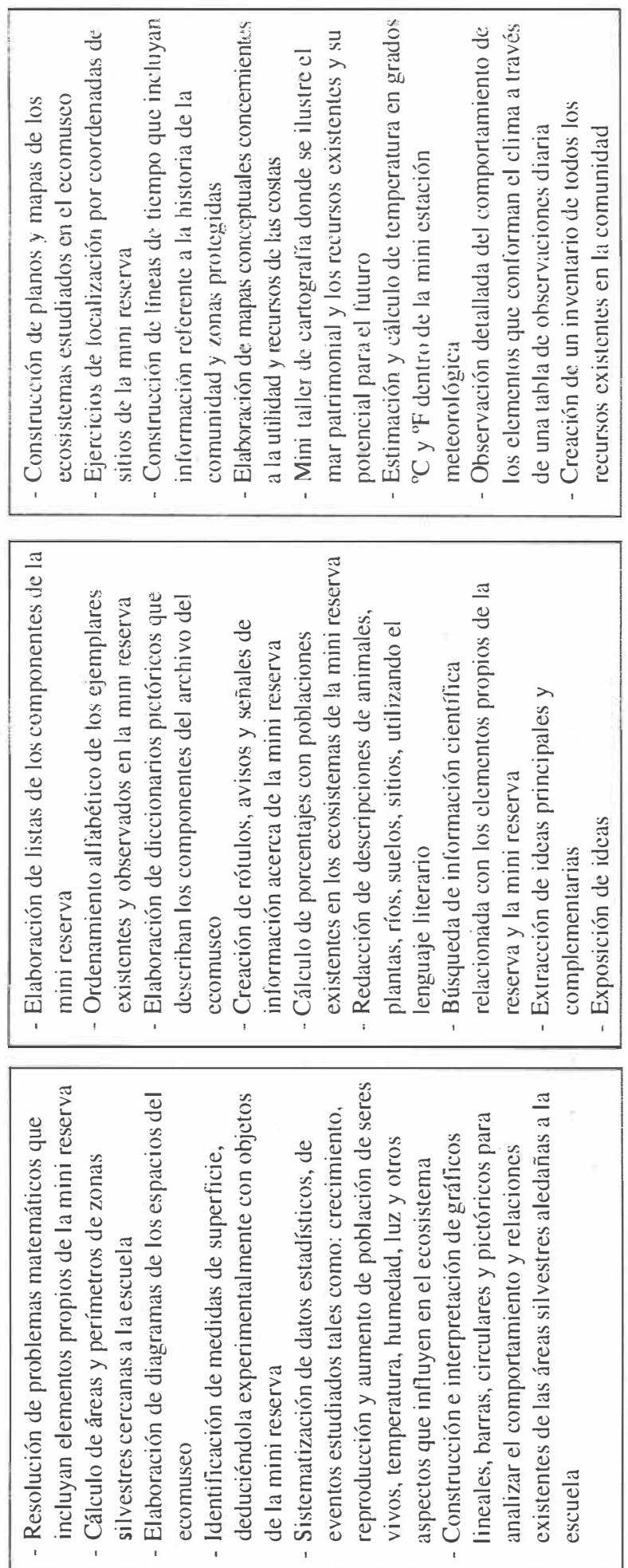

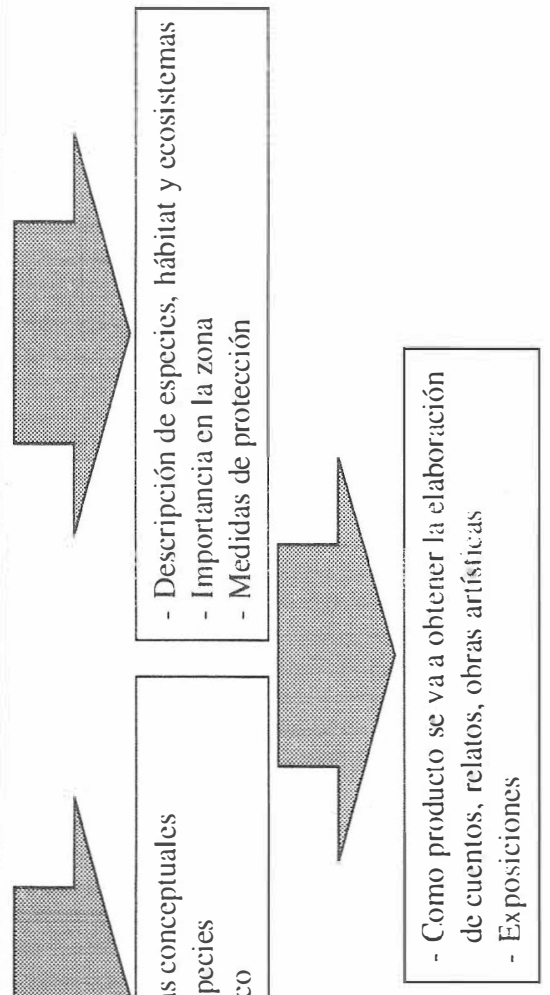

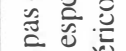
矛 导 ขั 을 든

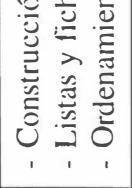




\section{Segundo Ciclo \\ Calendario Natural}

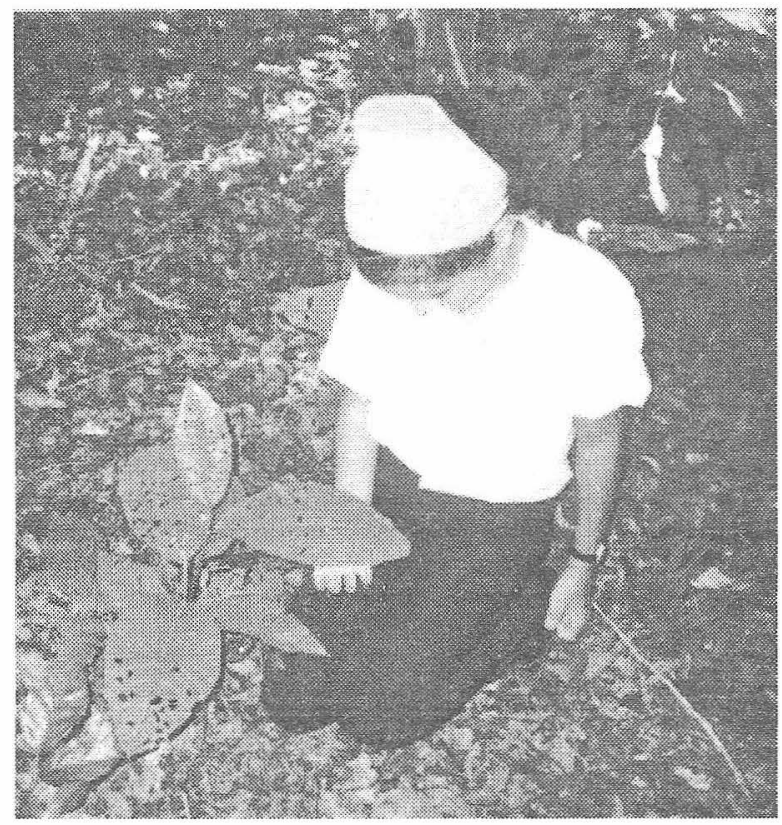

Grupo meta: I y II Ciclos

Áreas integradas:
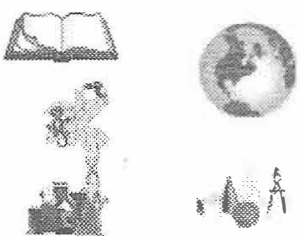

Espacios del ecomuseo:
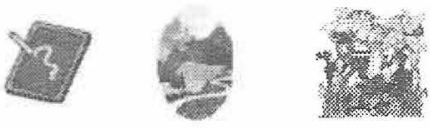

\section{Metodología:}

Participativa y observación activa.

\section{¿Por qué?}

La observación del contexto natural así como la interpretación de lo que se observe, es lo que persigue esta actividad.

Para ello se plantea la necesidad de realizar visitas de campo para que los niños y niñas detecten los cambios biológicos y físicos que se dan a su alrededor.

El concepto de tiempo y la noción de cómo transcurre es precisado por los estudiantes a partir de todos los elementos que los rodean y los cambios que dichos elementos sufren.

Dentro del ámbito rural se debe rescatar la información y conocimiento que los niños y niñas tienen acerca de su entorno, los seres vivos que le rodean, los fenómenos atmosféricos, el comportamiento del clima y del estado del tiempo, así como los cambios y modificaciones que se dan con los mismos representan un valioso arsenal de información que los estudiantes pueden utilizar como tema de estudio o investigación.

Al igual que las plantas, los animales obedecen cambios en su metabolismo que suceden a través de la variable tiempo. Tales cambios son observados, ya que las variaciones de color tamaño, predominancia, follaje (plantas) crías 
(animales) son evidentes y posiblemente estemos habituados a ello, pero no tenemos una conciencia temporal de cómo se dan dichas variaciones.

A través del calendario natural, cada día representa un descubrimiento de algo que sucede alrededor del niño, a partir de la observación de los indicios biológicos y físicos, se valoran las funciones vitales de seres vivos y su relación con el ecosistema al que pertenece.

\section{¿Cómo?}

1. Se debe contar con libretas o cuadernos que recopilen la información que los niños y niñas hayan observado. Para eso cada página representará un día, semana o mes (dependiendo de la especialidad según el grado).

2. En esta página o sección se anotarán todos los datos relacionados con:

a) Situación atmosférica. (Viento, la presencia o no de nubes, la temperatura, claridad, humedad).

b) Aparición de insectos, proliferación, desaparición- (Asociación biológica).

c) Indicios biológicos: mudas, huevos, nidos, plumas, dispersión de semillas, larvas, hojas secas, cortezas de árboles, aumento o disminución de follaje en las plantas, aparición de frutos, etc.

d) Aumento del caudal de ríos, quebradas, riachuelos, nacimientos y las consecuencias sobre el medio.

e) Tránsito de especies migratorias.

f) Interacción entre las poblaciones animales y el estado del tiempo.

g) Normancia o floración de las especies forestales y su duración de tales períodos.

h) Descripción del cambio de apariencia en mamíferos, aves y anfibios de la mini reserva.

3. Luegoen forma general se elaborará un calendario gigante (mural) donde en forma conjunta se anotarán los datos más significativos para cada día, semana o mes.

4. Cada nivel puede elaborar su calendario natural, según su capacidad y nivel de profundización, por ejemplo los niños de $3^{\circ}$ y $4^{\circ}$ grado las ilustraciones así como las muestras, son más significativas, mientras que en $5^{\circ}$ y $6^{\circ}$ se requiere de mayor descripción y explicación de los procesos observados.

5. El calendario natural permite a los niños y niñas reconocer la noción del tiempo como elemento presente en el desarrollo de la vida, así como detectar los signos propios de la misma. Les concede la oportunidad de comprar y relacionar distintos momentos y las características biológicas y físicas propias de dichos momentos. Pueden elaborar cuadros comparativos entre diversos 
meses e inclusive comparar el mismo mes, pero de distintos años para conocer e interpretar los cambios que se dan de un año a otro. Es en sí una bitácora recopiladora de un viaje biológico a través del tiempo.

6. Una vez que se han elaborado los calendarios y bitácoras (anotaciones) se puede ejecutar algunas acciones con los datos observados.
a) Elaboración de gráficos (con datos referentes a poblaciones, comportamiento climático u otros).
b) Creación de esquemas y diagramas.
c) Textos descriptivos de sitios, animales, plantas.
d) Adopción, estudio y seguimiento del crecimiento y desarrollo de alguna especie forestal.
e) Medición de periodos de algún proceso vital.
f) Ilustración representativa de cada mes del año.
g) Creación de cuentos, canciones y poemas. 


\section{INVENTARIO BIOLÓGICO DE LA MINIRESERVA (PLANTAS Y ANIMALES)}

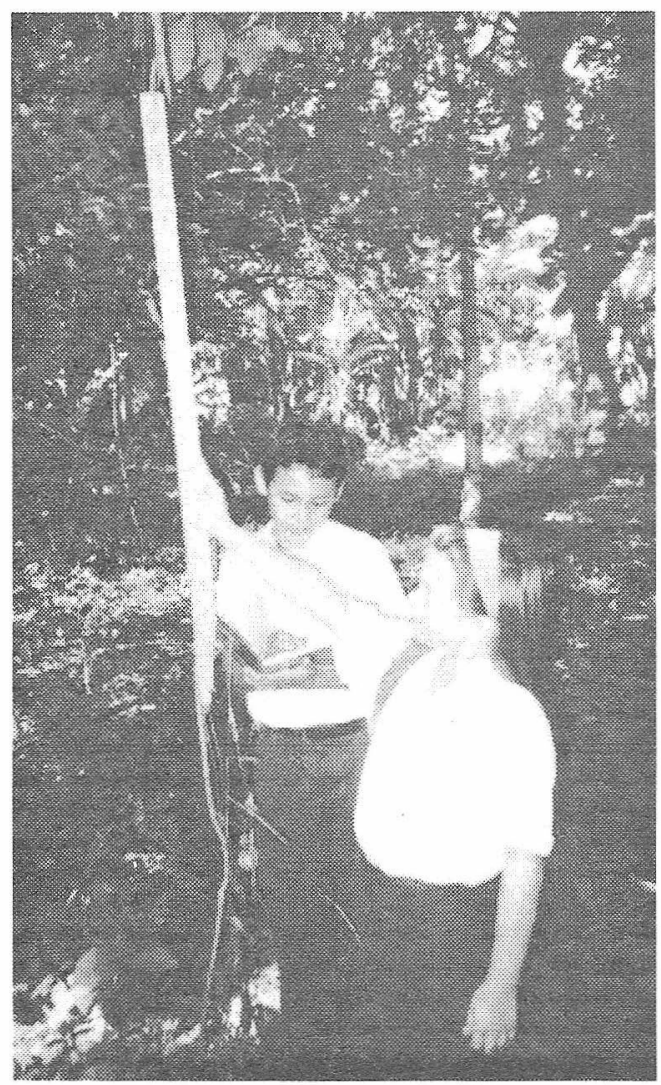

Grupo meta: I y II Ciclos

Áreas integradas:

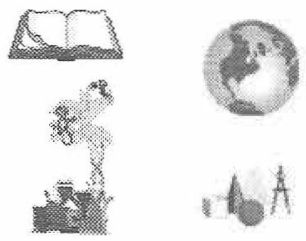

\section{Espacios del ecomuseo:}
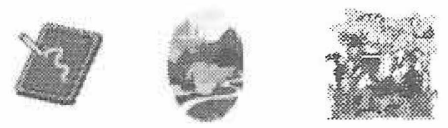

\section{Metodología:}

Participativa, grupal, experimental y activa.

\section{¿Por qué?}

Consiste en el aprendizaje a través de la observación detallada de fenómenos naturales que ocurren en la minireserva.

Para ello es necesario que los niños y niñas estén en contacto con todos los elementos existentes en ella para que resulten más significativos. Los estudiantes aprenden a observar y a describir lo que han observado usando sus propias palabras o nombres vernáculos ( de la región). A partir de ello pueden establecer conclusiones adecuadas en el ámbito grupal. Luego esas conclusiones y datos deben ser analizados, organizados de forma sistemática y creativa, para ello se recomienda el salón de clase y el museo escolar. 


\section{¿Cómo?}

1. Anterior a la visita a la minireserva se debe definir cuáles van a ser los aspectos que se estudiarán y de que forma se aplicarán las guías de campo. (anexo 1 y 2 ).

2. Con base en los niveles que participen se establecerá cuáles aspectos descriptivos pueden definir los niños y niñas.

3. Es importante mencionar que paralelamente a la observación que realicen los niños y niñas, se debe llevar una libreta en la que anoten en forma general sus apreciaciones.

4. La actitud y respeto hacia este espacio es muy importante, ya que propicia la percepción y permite la presencia de animales.

5. Se debe recordar que es una actividad de investigación práctica por lo tanto sus anotaciones tendrán valor en el proceso posterior a la visita.

6. Una vez que los estudiantes han recopilado la información que consideran pertinente, se procede a ordenar y analizar dicha información.

7. En las guías de campo, se deben ordenar y contabilizar las especies vistas (vegetales y animales).

8. Muchas de las especies requieren de ser observadas periódicamente para completar sus características descriptivas.

9. Los intervalos de tiempo varían de acuerdo a las necesidades cognitivas de los niños y niñas y su nivel. Asimismo los periódicos para la finalización de las guías dependen de la estación (seca, lluviosa o de transición) y de los temas que se desarrollen en clase, tanto en I ciclo como en el II ciclo. 


\section{JUGUEMOS AL ECÓLOGO}

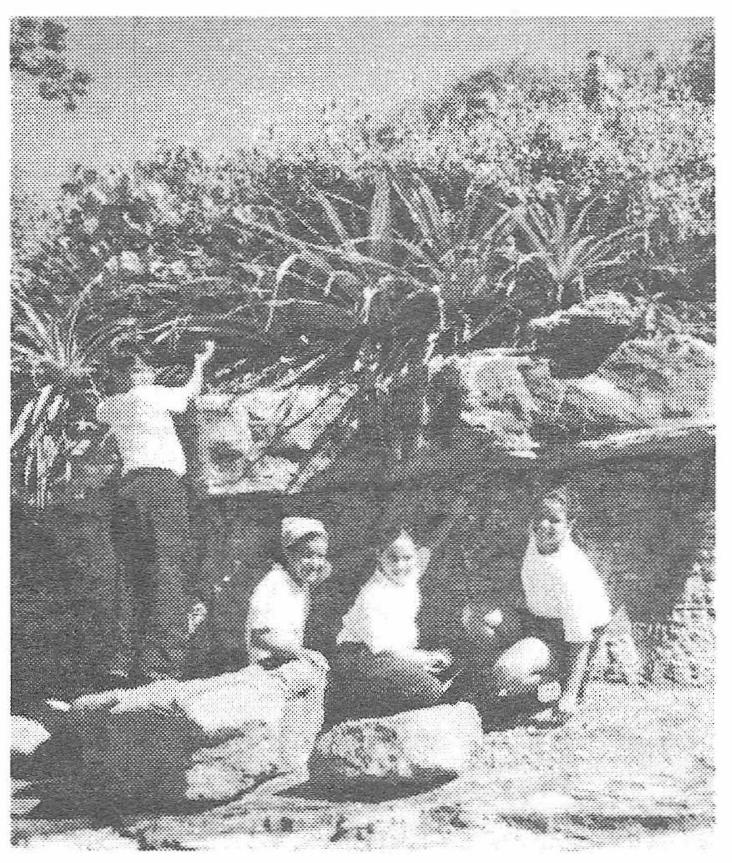

Grupo meta: I y II Ciclos

Áreas integradas:

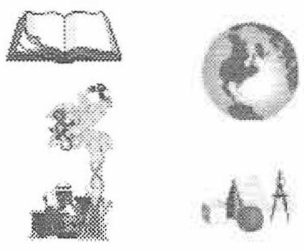

Espacios del ecomuseo:
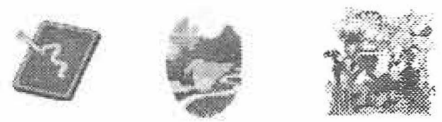

Metodología:

Participativa, experimental y observación.

\section{¿Por qué?}

La preferencia de los temas científicos por parte de los niños y las niñas permite desde cualquier punto de vista, un enriquecimiento que apoya el aprendizaje.

La visita a zonas protegidas proporciona oportunidades de aprendizaje significativo, ya que son espacios que en la mayoría de los casos impresionan y despiertan el interés de los niños y niñas.

\section{¿Cómo?}

1. Se organiza con anterioridad la visita o excursión a una zona protegida cercana al centro educativo.

2. Se subdivide el grupo o grupos de acuerdo a las edades y temas a investigar (por grados, por afinidad, etc.).

3. Se debe tener claro cuál es el tipo de actitud y conducta durante la excursión para darle a la actividad un clima de armonía y relación con la naturaleza. 
Durante la exploración los estudiantes recopilan la información relevante acerca de la zona en estudio:
a) Información general.
b) Recursos existentes.
c) Descripción de especies animales y vegetales.
d) Ilustraciones.
e) Conclusiones.
f) Aportes al mural ecológico.

Resolución de la actividad: «Explorando nuestras zonas protegidas». (Anexo 4). Puesta en común donde se analicen los datos recopilados, se comparan y se sintetizan. 


\section{EXPLORANDO LA COSTA}

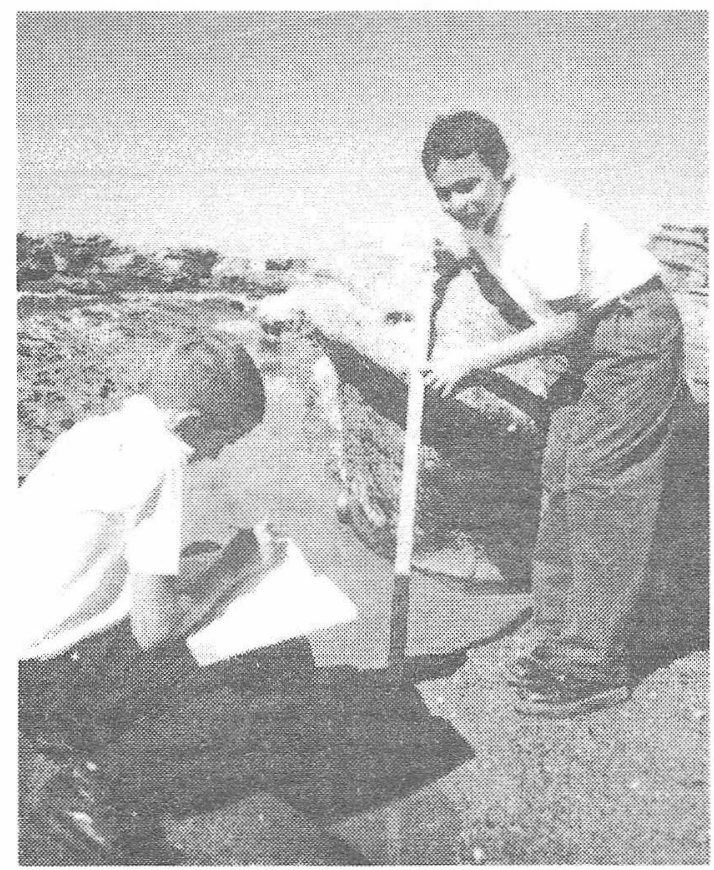

Grupo meta: I y II Ciclos

Áreas integradas:

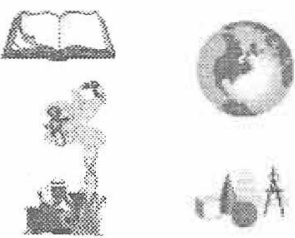

Espacios del ecomuseo:
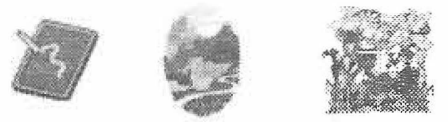

Metodología:

Participativa, experimental e investigativa.

\section{¿Por qué?}

El mar es uno de los recursos didácticos más ricos de explotar en las comunidades que se encuentran en la costa o cercanas a ella. Ofrece un gran atractivo, ya que permite a los estudiantes apreciar uno de los hábitats más rigurosos para los seres vivos. Dos veces al día estas criaturas que van cubiertas por el agua y cuando la marea se retira, se adaptan a la falta de humedad. Se hallan expuestos al calor, al frío y a las fuertes olas.

Las condiciones son cambiantes. Cada marea trae consigo una nueva provisión de alimento en forma de criaturas marinas microscópicas.

Existen un sin fin de especies, pero a través de la observación y las anotaciones se hace más fácil comprender y apreciar su presencia en dichos sitios.

La costa es uno de los lugares más divertidos para los niños y niñas. Siempre hay algo que ver y cada elemento que estudien los niños proporciona un aprendizaje más significativo y armonioso. 


\section{¿CÓMO?}

1. Para descubrir qué animales viven en la costa, especialmente en los niveles bajos, los estudiantes junto con el maestro siguen la marea que se retira de la playa, rocas y arrecifes.

2. Los estudiantes en grupos por nivel elaboran un mapa del sector comprendido entre la mare baja hasta el sector de salpicado, durante la marea alta. En dicho mapa se debe establecer qué puntos son propensos a llenarse, humedecerse o secarse durante los diferentes momentos del día.

3. En el mapa se trazan unas coordenadas para ubicar los seres vivos que se localizan en cada sector. Muchos animales se esconden en grietas y bajo los arrecifes. Estos deben registrarse en el mapa y en el archivo del ecomuseo y así compararse con los animales de otras áreas.

4. Dentro del mapa se debe dejar claro los diversos niveles de mareas e incluso la zona de salpicado en la cual habitan otra cantidad considerable de seres vivos.

5. A partir de los pasos anteriores, los estudiantes pueden construir relatos de sus experiencias en la zona de mareas y realizar descripciones orales o escritas que enriquezcan el material del museo y exponer al igual que otros, sus mapas de la costa.

6. Para dicha actividad se requiere mucho orden y respeto, ya que se apreciarán las especies en silo por lo cual la intervención en el ecosistema visitado debe ser mínima. 


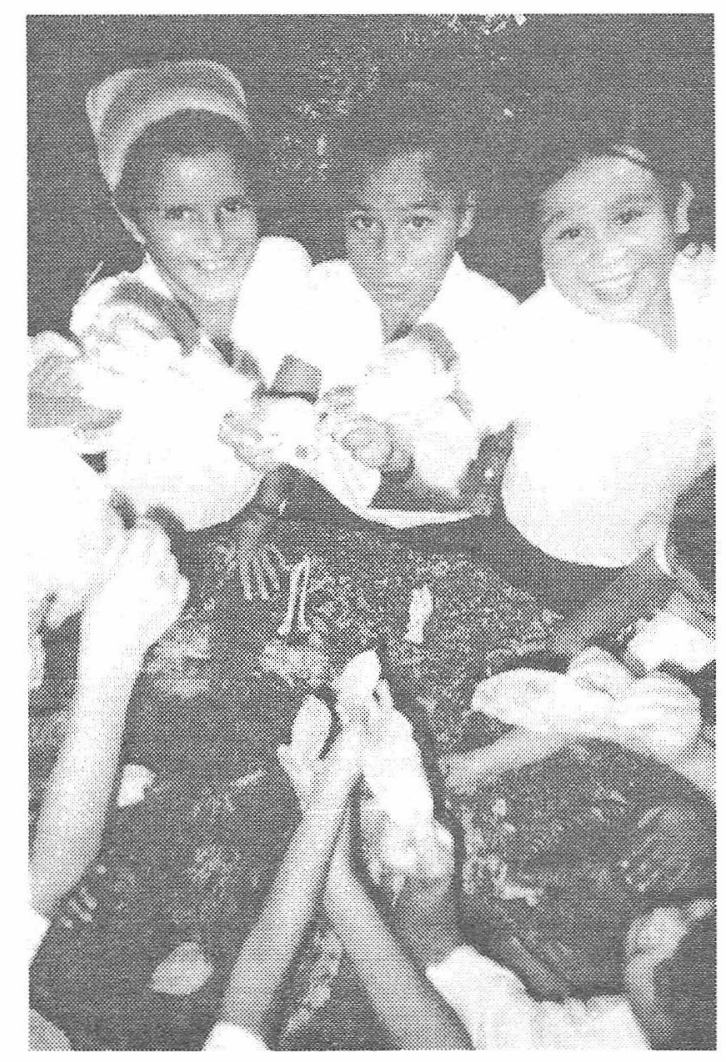

Grupo meta: I y II Ciclos

\section{Áreas integradas:}
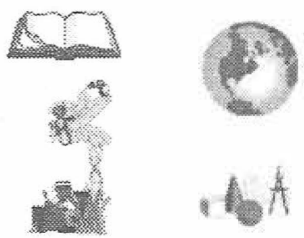

\section{Espacios del ecomuseo:}
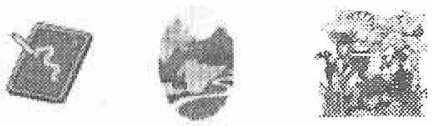

\section{Metodología:}

Participativa, experimental y activa.

\section{¿Por qué?}

Los bosques aledaños a la escuela representan un valioso recurso, ya que ofrece al pequeño investigador una gran cantidad de información viviente. Esta información facilita el aprendizaje a partir de la observación e interpretación de los fenómenos que se den en el entorno de los estudiantes.

Por otra parte, el conocimiento informal de los habitantes de la comunidad con respecto al bosque y sus recursos es muy rico, pues a través de sus experiencias y costumbres han podido recopilar un saber útil. Este conocimiento debe rescatarse y asegurar en alguna medida una relación entre las actuales generaciones y el saber popular propio de cada región. Usos, aplicaciones y características de las plantas son datos que desarrollan en los niños un sentido de arraigo y pertenencia hacia lo propio. 
1. A través de las excursiones y visitas a la mini reserva los estudiantes recopilan muestras de plantas y árboles existentes en dicha zona.

2. Al hablar de «muestras» se debe entender partes de la planta como hojas, trozos de cortezas, semillas, flores, frutos y trozos de ramas.

3. Una vez que se han clasificado las distintas partes se procede a realizar una plenaria en donde los niños y niñas expresen sus apreciaciones acerca de las especies identificadas en la mini reserva.

4. Para tener unaidea exacta sobre el lugar donde se encuentran las especies vegetales. se elabora un pequeño mapa mural y se sitúan los árboles y plantas estudiadas.

5. Con las hojas recolectadas se procede al secado y almacenado para la conformación del herbario.

6. Cada planta que es representada en el herbario debe poseer una ficha de información en el archivo de Ecomuseo donde se anoten diferentes aspectos tales como: altura, espesor y forma del tallo. Su apariencia en los colores. forma y disposición del follaje, floración y otros son elementos que deben ser considerados para describir el espécimen.

7. Una vez realizado el secado de las hojas se elabora un texto para exponer cada muestra con información recolectada por los niños en sus hogares y comunidad Algunas de estas premisas son:

a) El uso que le dan los vecinos de la comunidad a esa planta.

b) Propiedades medicinales que se han detectado.

c) Otros nombres con los que se conoce en la zona.

d) Forma de reproducción.

e) Anécdotas de los campesinos.

8. El herbario forestal es un proyecto de mucho interés para los estudiantes porque los induce a observar y expresar sus ideas. Brinda posibilidades infinitas a la hora de participar en grupos, parejas o hasta como proyecto individual en grados superiores, cada hoja representa una experiencia cognitiva que impulsa otros niveles en el interés de los niños y las niñas. 


\section{ANEXOS \\ Anexo $\mathrm{N}^{\circ} 1$ \\ GUÍA DE CAMPO}

Inventario de especies de animales de la Minireserva

\begin{tabular}{|c|c|c|c|c|c|}
\hline Nombre común & Nombre científico & Inportancia Ecolígica & $\begin{array}{l}\text { Tipo (Mamifero, ave, reptil. } \\
\text { anfibio o insecto) }\end{array}$ & Híbitat & Háhitos \\
\hline & & & & & \\
\hline & & & & & \\
\hline & & & & & \\
\hline & & & & & \\
\hline & & & & & \\
\hline & & & & & \\
\hline$=$ & & & & & \\
\hline & & & & & \\
\hline & & & & & \\
\hline
\end{tabular}

Tomado del Programa Acción Ambiental (Reserva Nal. Absoluta Cabo Blanco)

\section{Anexo $\mathrm{N}^{\circ} 2$ \\ GUÍA DE CAMPO}

Inventario de especies de plantas de la Minireserva

\begin{tabular}{|c|c|c|c|c|c|}
\hline Nombre común & Nombre científico & Importancia Ecoltigica & Mes de floración & $\begin{array}{l}\text { Mes de } \\
\text { fruclificación }\end{array}$ & Color de ther \\
\hline & & & & & \\
\hline & & & & & \\
\hline & & & & & \\
\hline & & & & & \\
\hline & & & & & \\
\hline & & & & & \\
\hline & & & & & \\
\hline & & & & & \\
\hline & & & & & \\
\hline
\end{tabular}

Tomado del Programa Acción Ambiental (Reserva Nal. Absoluta Cabo Blanco) 


\section{Anexo $\mathrm{N}^{\bullet} 3$ \\ INVESTIGACIÓN}

Anotar en el cuadro las características de las hojas de las plantas que se investigan.

\begin{tabular}{|c|c|c|c|c|c|}
\hline Nombre común & Olor & $\begin{array}{l}\text { Estructura: Espinas. } \\
\text { nelos. atrun }\end{array}$ & $\begin{array}{l}\text { Textura: suave. } \\
\text { unra. gruesa }\end{array}$ & Tamaño (cm) & $\begin{array}{l}\text { Tipe: Simple } \\
\text { o compunuestia }\end{array}$ \\
\hline & & & & & \\
\hline & & & & & \\
\hline & & & & & \\
\hline & & & & & \\
\hline & & & & & \\
\hline & & & & & \\
\hline & & & & & \\
\hline & & & & & \\
\hline & & & & & \\
\hline
\end{tabular}

Tomado del Programa Acción Ambiental (Reserva Nal. Absoluta Cabo Blanco)

\section{Anexo $\mathrm{N}^{\circ} 4$ \\ EXPLORANDO NUESTRAS ZONAS PROTEGIDAS}

Actividad \# 1

A. Organicemos con ayuda de nuestra maestra una excursión a una área silvestre protegida que se encuentre cerca de nuestro lugar de residencia o centro educativo.

B. Investigación.

1. Información general.

Nombre del área silvestre

Localización

Hectáreas que comprende

Año en que se declaró zona protegida

Provincia Cantón 
2. ¿Cuál fue el propósito o motivo al declarar el área como zona protegida?

3.- Llene el siguiente cuadro con la información que se solicita.

Recursos protegidos en el área de visita

\begin{tabular}{|c|c|c|}
\hline Especies animales & Especies vegetales & Minerales \\
\hline & & \\
\hline & & \\
\hline & & \\
\hline & & \\
\hline & & \\
\hline & & \\
\hline & & \\
\hline & & \\
\hline & & \\
\hline & & \\
\hline & & \\
\hline & & \\
\hline & & 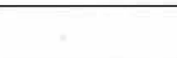 \\
\hline & & \\
\hline & & \\
\hline & & \\
\hline & & \\
\hline & & \\
\hline & & \\
\hline & & \\
\hline & & \\
\hline & & \\
\hline
\end{tabular}


4.- ¿Cuáles de las especies animales o vegetales están en peligro de extinción?

\begin{tabular}{|l|l|}
\hline Especies animales & Especies regetales \\
\hline & \\
\hline
\end{tabular}

5.- Escriba algunas razones por las cuales las especies anteriormente están a punto de desaparecer.

6.- Imagine representando por medio del dibujo lo que ocurriría con la ausencia de los animales y plantas que están en peligro de extinción.
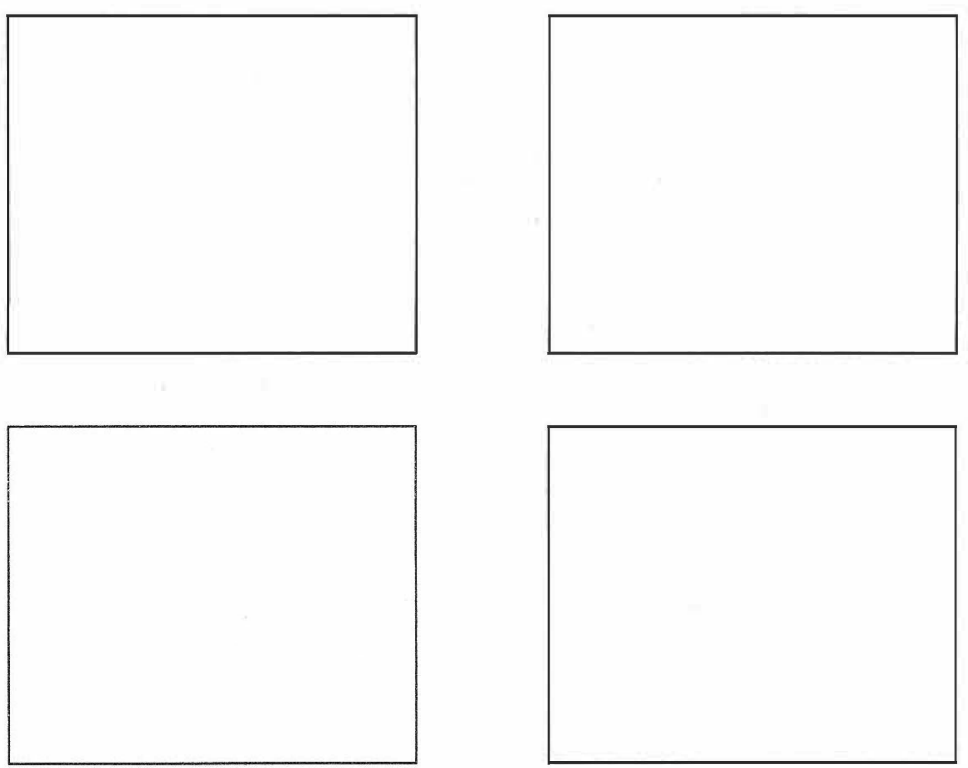
7.- ¿Qué opinan los funcionarios del Parque o de la Reserva acerca de la protección de plantas y animales silvestres?

8.- Investigue con personas de la comunidad acerca de la importancia que tiene un Parque Nacional o una Reserva para proteger las plantas y animales silvestres.

Redacte su resumen: 